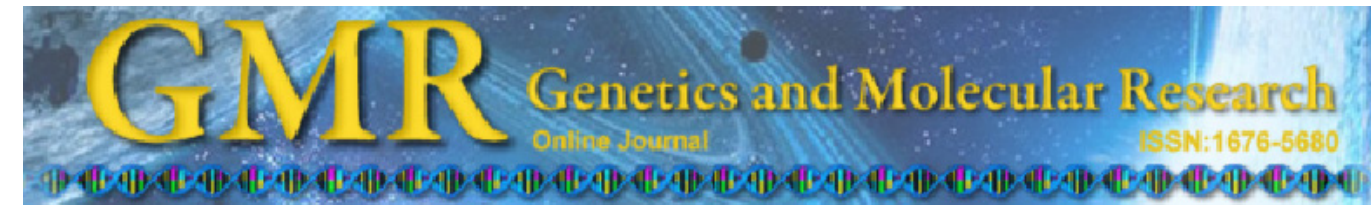

\title{
Analysis of genetic distance by SSR in waxy maize
}

\author{
R.H. Yu, Y.L. Wang, Y. Sun and B. Liu \\ College of Agronomy, Jilin Agricultural University, Changchun, China \\ Corresponding author: Y.L. Wang \\ E-mail: yuronghai2011@163.com
}

Genet. Mol. Res. 11 (1): 254-260 (2012)

Received May 2, 2011

Accepted October 28, 2011

Published February 3, 2012

DOI http://dx.doi.org/10.4238/2012.February.3.5

\begin{abstract}
We examined the genetic diversity of 80 inbred waxy maize lines using 22 SSR molecular markers that could be used to achieve heterosis in waxy maize. Eighty inbred waxy maize lines with different phenotypes, 40 yellow, 25 white, 13 black, and two red lines were analyzed by SSR molecular marker fingerprint and cluster analysis. Using a standard genetic distance of 0.55 , the 80 waxy maize inbred lines were clustered into nine groups. Among them, group II, group V, groups VII and VIII, and group IX were divided into three subgroups at a genetic distance of 0.46 , into two subgroups at 0.49 , into two subgroups at 0.46 , and into four subgroups at 0.493 , respectively. All but one of the yellow waxy maize inbred lines were clustered in groups VI, VII, VIII, and IX. Group IX (30 lines) contained 28 yellow lines; the other 11 yellow lines were distributed among groups VI, VII and VIII. Among the 25 white lines, 21 were clustered in groups III, V, VI and the third subgroup of group II. The black line N72 was in a group of its own. The black lines N75, N76 and N78 were distributed in groups VII, VIII and IX, respectively. The other nine black lines were clustered in group II. The red lines were distributed in the second subgroup of group II and there was no difference in genetic distance between them. In conclusion, there were considerable genetic differences among waxy maize inbred lines of different colors. The mean genetic distance of inbred lines of the same color was significantly less than that of lines
\end{abstract}


of different colors. Therefore, we concluded that it was more accurate to determine the difference between the populations using the highly stable DNA genetic markers.

Key words: Waxy maize; SSR; Genetic distance

\section{INTRODUCTION}

Waxy maize, a valuable germplasm resource originating from China, is a selective breeding variety with endosperm mutation controlled by the recessive waxy gene (wxwx) and recessive homozygote (wxwx) can lead to almost $100 \%$ of amylopectin in the endosperm (Rief et al., 2003a; Liu et al., 2005; Yao et al., 2007). Through several centuries of variation and selection, there is abundant genetic diversity in grain color, yield, quality, and resistance of waxy maize. Previous studies mainly focused on the quality, physiology and biochemistry, cultivation, nutritional and individual agronomic traits in waxy maize (Rief et al., 2003b; Xia et al., 2004). With the rapid development of molecular biology, molecular markers have been widely used in the study of normal maize (Nei and Li, 1979; Novy et al., 1994; Wang et al., 1994; Smith et al., 1984, 1997; Senior et al., 1998; Wu et al., 1999; Shehata et al., 2009). However, reports about genetic diversity of waxy maize at the molecular level are relatively few. Reif et al. (2003a,b) studied the heterosis of tropical and subtropical maize. Liu et al. (2005) have carried out the study of the waxy local varieties from Guizhou and Yunnan using SSR markers. Yao et al. (2007) have analyzed the diversities of waxy maize lines by SSR markers in Southwest China. There is a need to understand the genetic relationship among waxy maize germplasms at the molecular level in order to improve waxy maize breeding.

The purpose of this study was to reveal the genetic diversity in 80 waxy maize inbred lines, 40 yellow, 25 white, 13 black, and 2 red lines, using 22 SSR molecular markers. This is the first study of the molecular diversity in waxy maize by SSR markers. The clustering results can reveal the genetic relationship at the molecular level and further provide theoretical understanding for the application of heterosis in waxy maize.

\section{MATERIAL AND METHODS}

\section{Materials}

A total of 80 waxy maize inbred lines (including 40 yellow, 25 white, 13 black, and 2 red waxy maize inbred lines) were provided by the special maize group in Jilin Agricultural University (Table 1).

\section{Genomic DNA extraction}

Genomic DNA of waxy maize inbred lines was extracted from the leaves using a modified cetyltrimethylammonium bromide $(\mathrm{CTAB})$ procedure. The concentrations and quality of DNA were measured in a Beckman DU-65 spectrophotometer. DNA was diluted to 20 $\mathrm{ng} / \mu \mathrm{L}$ for use. 
Table 1. The 80 waxy maize inbred lines and their codes.

\begin{tabular}{|c|c|c|c|c|c|c|c|}
\hline Code & Inbred line & Code & Inbred line & Code & Inbred line & Code & Inbred line \\
\hline N01 & YW1 & N21 & YW21 & N41 & WW41 & N61 & WW61 \\
\hline N02 & YW2 & $\mathrm{N} 22$ & YW22 & N42 & WW42 & N62 & WW62 \\
\hline N03 & YW3 & N23 & YW23 & N43 & WW43 & N63 & WW63 \\
\hline N04 & YW4 & N24 & YW24 & N44 & WW44 & N64 & WW64 \\
\hline N05 & YW5 & $\mathrm{N} 25$ & YW25 & N45 & WW45 & N65 & WW65 \\
\hline N06 & YW6 & N26 & YW26 & N46 & WW46 & N66 & BW66 \\
\hline N07 & YW7 & $\mathrm{N} 27$ & YW27 & N47 & WW47 & N67 & BW67 \\
\hline N08 & YW8 & N28 & YW28 & N48 & WW48 & N68 & BW68 \\
\hline N09 & YW9 & N29 & YW29 & N49 & WW49 & N69 & BW69 \\
\hline N10 & YW10 & N30 & YW30 & N50 & WW50 & N70 & BW70 \\
\hline N11 & YW11 & N31 & YW31 & N51 & WW51 & N71 & BW71 \\
\hline N12 & YW12 & N32 & YW32 & N52 & WW52 & N72 & BW72 \\
\hline N13 & YW13 & N33 & YW33 & N53 & WW53 & N73 & BW73 \\
\hline N14 & YW14 & N34 & YW34 & N54 & WW54 & N74 & BW74 \\
\hline N15 & YW15 & N35 & YW35 & N55 & WW55 & N75 & BW75 \\
\hline N16 & YW16 & N36 & YW36 & N56 & WW56 & N76 & BW76 \\
\hline N17 & YW17 & N37 & YW37 & N57 & WW57 & N77 & BW77 \\
\hline N18 & YW18 & N38 & YW38 & N58 & WW58 & N78 & BW78 \\
\hline N19 & YW19 & N39 & YW39 & N59 & WW59 & N79 & RW79 \\
\hline $\mathrm{N} 20$ & YW20 & N40 & YW40 & N60 & WW60 & N80 & RW80 \\
\hline
\end{tabular}

$\mathrm{YW}=$ yellow waxy maize inbred lines; $\mathrm{WW}=$ white ones; $\mathrm{BW}=$ black ones; $\mathrm{RW}=$ red ones.

\section{SSR analysis}

In this assay, 22 pairs of core primers distributed in the 10 chromosomes of maize were used (Table 2). Each $20 \mu \mathrm{L}$ PCR mixture contained 40 ng genomic DNA, $150 \mu \mathrm{M}$ dNTPs, 2.5 $\mathrm{mM} \mathrm{MgCl} 2,0.25 \mu \mathrm{M}$ primers, $2.0 \mu \mathrm{L} 10 \mathrm{X}$ Taq buffer and $1 \mathrm{U}$ Taq polymerase. Samples were subjected to the following thermal profile: 5 min of denaturation at $94^{\circ} \mathrm{C}, 35$ cycles of $40 \mathrm{~s}$ of denaturation at $94^{\circ} \mathrm{C}, 35 \mathrm{~s}$ of annealing at $68^{\circ} \mathrm{C}$ and $45 \mathrm{~s}$ of elongation at $72^{\circ} \mathrm{C}$, with a final elongation step of $10 \mathrm{~min}$ at $72^{\circ} \mathrm{C}$. Amplification products were separated on $6.0 \%$ polyacrylamide gels and gels were silver stained after electrophoresis.

Table 2. SSR core primers used for the waxy maize inbred lines in this study.

\begin{tabular}{rccccr}
\hline No. & Primer & Chromosome location & No. & Primer & Chromosome location \\
\hline 1 & N01 & 1.03 & 12 & N93 & 10.07 \\
2 & N73 & 2.02 & 13 & N137 & 1.10 \\
3 & N81 & 3.09 & 14 & N159 & 2.07 \\
4 & N67 & 4.00 & 15 & N133 & 3.00 \\
5 & N05 & 5.03 & 16 & N131 & 4.06 \\
6 & N88 & 5.06 & 17 & N184 & 5.05 \\
7 & N64 & 6.00 & 18 & N193 & 6.01 \\
8 & N98 & 7.02 & 19 & N136 & 7.06 \\
9 & N201 & 8.05 & 20 & N119 & 8.08 \\
10 & N96 & 8.03 & 21 & N58 & 9.01 \\
11 & N92 & 9.03 & 22 & N20 & 10.04 \\
\hline
\end{tabular}

\section{Data analysis}

According to the results of molecular markers (Figure 1), the SSR bands were scored manually and analyzed as binary data, with 1 representing the presence and 0 representing the absence of a band at a particular location in each lane. The genetic similarity coefficient 
(GS) and genetic distance (GD) between the waxy maize inbred lines were calculated according to the Nei's formula of $\mathrm{GSij}=2 \mathrm{Nij} /(\mathrm{Ni}+\mathrm{Nj})$ and $\mathrm{GDij}=1-\mathrm{GSij}$, where $N i j$ represents the number of common bands between the $\mathrm{i}$ and $\mathrm{j}$ inbred lines; $\mathrm{Ni}$ and $\mathrm{Nj}$ represent the total number of bands of the $i$ and $j$ inbred lines, respectively. Data were analyzed with the DPS 3.0 software. Cluster analysis of matrix values was performed by the unweighted pair group method with arithmetic averages (UPGMA). Dendrograms showing the relationships between SSR patterns were generated from these matrices using the Tree-plot program.

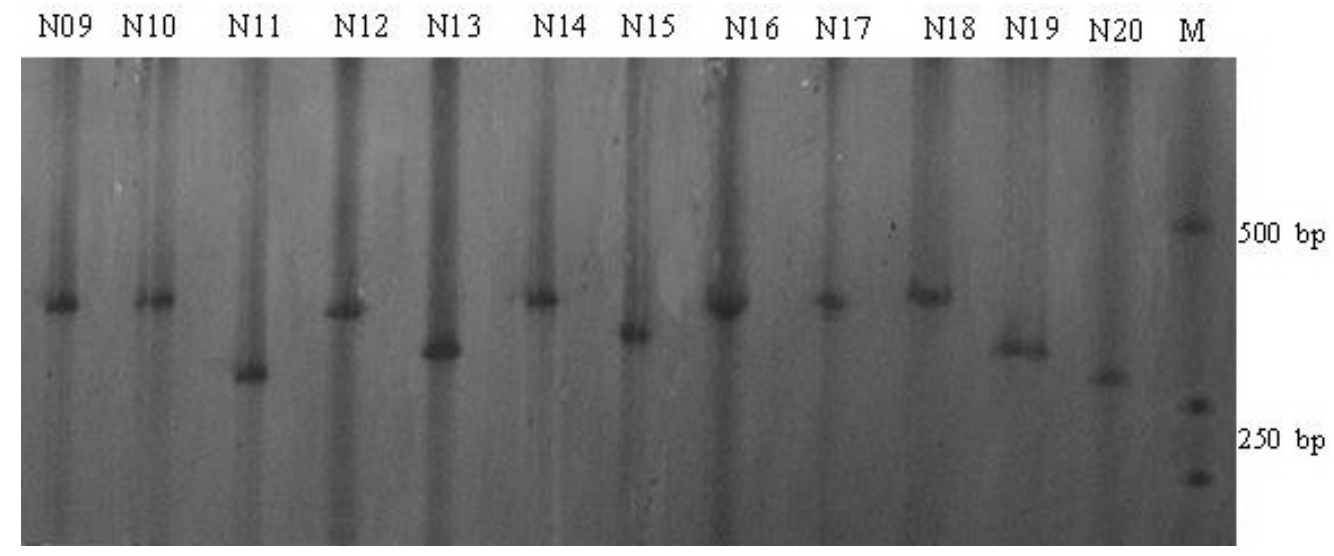

Figure 1. Amplification results of the 80 waxy maize inbred lines. The primers N159 and N173 were at sites of 2.07 and 2.02. Lane $M=$ Molecular marker.

\section{RESULTS}

\section{Genetic distances among the 80 waxy maize inbred lines by SSR analysis}

Nei's GD among the 80 waxy maize inbred lines were between 0.050 and 0.620 in this study. The 22 pairs of SSR core primers were distributed in 10 chromosomes of maize from 80 isolates, 60 polymorphic alleles were amplified by 22 SSR primer pairs, and the polymorphic alleles ranged from 2 to 4 per locus, with an average of 2.73 (Table 3).

\section{Clustering results for the 80 waxy maize inbred lines}

The 80 waxy maize inbred lines were clustered into nine SSR groups (SSRGs) at the GD level of 0.55, using the UPGMA method based on the genetic distance matrix (Figure 2, Table 4). The dendrogram showed that SSRG II at the 0.46 GD level separated into three subgroups, SSRG V at the 0.50 GD level into two subgroups, SSRG VII at the 0.46 GD level into two subgroups, SSRG VIII at the 0.46 GD level into two subgroups, and SSRG IX at the 0.44 GD level into four subgroups (Figure 2, Table 4).

The SSR clustering distribution results were as follows. Group I included N12. Group II with three subgroups included N69, N80, N79, N73, N71, N70, N74, N68, N67, N65, N63, N61, N66, and N52. Group III included N50 and N48. Group IV included N72. Group V with two subgroups included N64, N59, N58, N46, N44, N62, N57, N54, N53, 
N60, N56, N55, and N43. Group VI included N16, N42, N16, and N41. Group VII with two subgroups included N40, N36, N25, N39, N37, N78, N77, and N09. Group VIII with two subgroups included N76, N45, N47, N11, N49, N07, and N06. Group IX with 4 subgroups included N75, N51, N30, N13, N14, N29, N28, N23, N33, N38, N19, N27, N34, N35, N18, N15, N10, N26, N05, N24, N22, N32, N31, N21, N03, N04, N02, N08, N20, and N01 (Table 4).

Table 3. Number of polymorphic alleles and product size amplified by SSR primers.

\begin{tabular}{|c|c|c|c|c|c|}
\hline Code & Primer & Chromosome location & Repeat motif & Polymorphic alleles & Product size (bp) \\
\hline 1 & N01 & 1.03 & $(\mathrm{AG})_{17}$ & 3 & $>533$ \\
\hline 2 & N73 & 2.02 & $(\mathrm{AG})_{16}$ & 4 & $350-560$ \\
\hline 3 & N81 & 3.09 & $(\mathrm{AG})_{18}^{16}$ & 2 & $400-533$ \\
\hline 4 & N67 & 4.00 & $(\mathrm{GT})_{4}(\mathrm{GA})_{6}$ & 2 & $>533$ \\
\hline 5 & N05 & 5.03 & $(\mathrm{AG})_{42}$ & 3 & $>533$ \\
\hline 6 & N88 & 5.06 & $(\mathrm{CT})_{17}$ & 4 & $230-480$ \\
\hline 7 & N64 & 6.00 & $(\mathrm{TA})_{10}$ & 2 & $>533$ \\
\hline 8 & N98 & 7.02 & $(\mathrm{CT})_{25}$ & 4 & $533-600$ \\
\hline 9 & N201 & 8.05 & $(\mathrm{AG})_{35}$ & 2 & $533-550$ \\
\hline 10 & N96 & 8.03 & $(\mathrm{AG})_{33}^{33}$ & 4 & $222-469$ \\
\hline 11 & N92 & 9.03 & $(\mathrm{AG})_{18}^{33}$ & 2 & $>533$ \\
\hline 12 & N93 & 10.07 & $(\mathrm{CA})_{32}$ & 2 & $>533$ \\
\hline 13 & N137 & 1.1 & $(A G)_{21}^{32}$ & 2 & $>533$ \\
\hline 14 & N159 & 2.07 & $(\mathrm{GA})_{39}$ & 3 & $168-222$ \\
\hline 15 & N133 & 3.00 & $(\mathrm{AG})_{22}$ & 3 & $>533$ \\
\hline 16 & N131 & 4.06 & $(\mathrm{AG})_{17}^{22}$ & 3 & $>533$ \\
\hline 17 & N184 & 5.05 & $(\mathrm{CT})^{17}$ & 3 & $312-469$ \\
\hline 18 & N193 & 6.01 & $(A G)_{17}^{16}$ & 2 & $132-350$ \\
\hline 19 & N136 & 7.06 & $(\mathrm{TG})_{12}^{17}$ & 3 & $290-350$ \\
\hline 20 & N119 & 8.08 & $(\mathrm{AG})_{16}$ & 3 & $469-480$ \\
\hline 21 & N58 & 9.01 & $(\mathrm{AG})_{14}$ & 2 & $220-230$ \\
\hline 22 & N20 & 10.04 & $(\mathrm{AC})_{24}$ & 2 & $>533$ \\
\hline Total & & & & 60 & \\
\hline Average & & & & 2.73 & \\
\hline
\end{tabular}

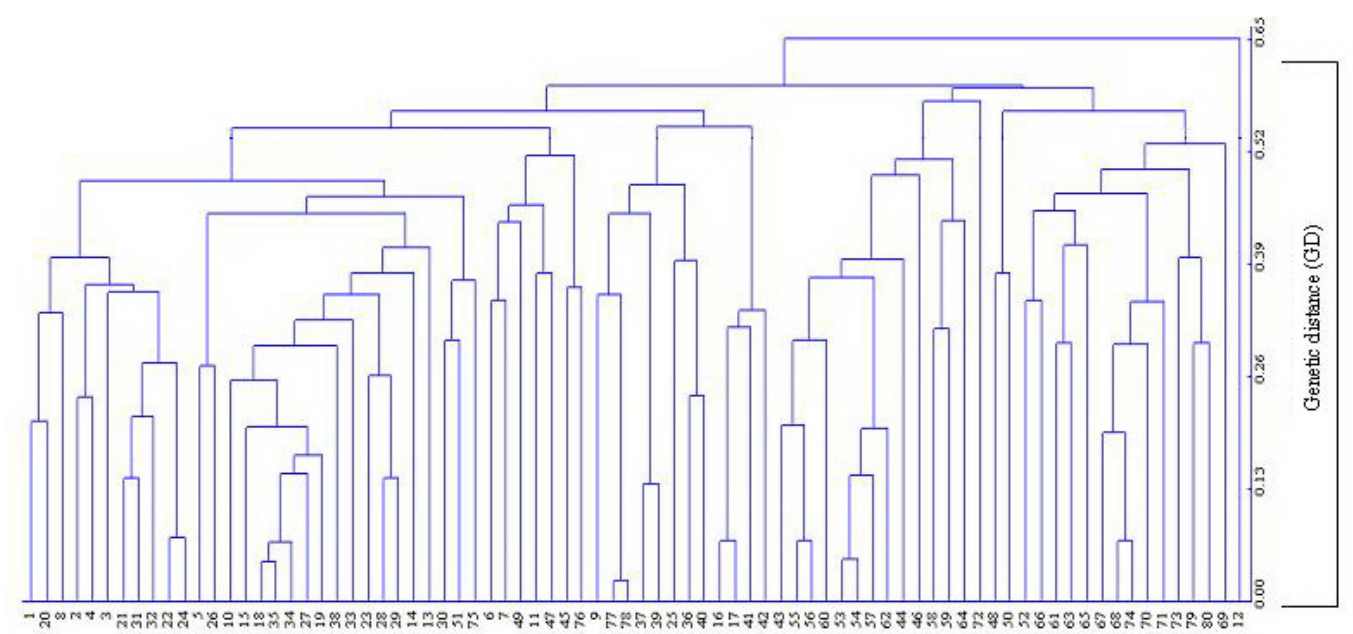

Figure 2. SSR clustering dendrograms of 80 waxy maize inbred lines.

Genetics and Molecular Research 11 (1): 254-260 (2012)

CFUNPEC-RP www.funpecrp.com.br 
Table 4. SSR clustering results of the 80 waxy maize inbred lines.

\begin{tabular}{lll}
\hline Group & Subgroup & Inbred lines \\
\hline I & & $\mathrm{N} 12$ \\
$\alpha$ & $\alpha 1$ & $\mathrm{~N} 69$ \\
& $\alpha 2$ & $\mathrm{~N} 80, \mathrm{~N} 79, \mathrm{~N} 73$ \\
$\beta$ & $\alpha 3$ & $\mathrm{~N} 71, \mathrm{~N} 70, \mathrm{~N} 74, \mathrm{~N} 68, \mathrm{~N} 67, \mathrm{~N} 65, \mathrm{~N} 63, \mathrm{~N} 61, \mathrm{~N} 66, \mathrm{~N} 52$ \\
$\chi$ & & $\mathrm{N} 72$ \\
$\delta$ & $\delta 1$ & $\mathrm{~N} 64, \mathrm{~N} 59, \mathrm{~N} 58$ \\
& $\delta 2$ & $\mathrm{~N} 46, \mathrm{~N} 44, \mathrm{~N} 62, \mathrm{~N} 57, \mathrm{~N} 54, \mathrm{~N} 53, \mathrm{~N} 41$ \\
$\varepsilon$ & $\phi 1$ & $\mathrm{~N} 40, \mathrm{~N} 36, \mathrm{~N} 25$ \\
$\phi$ & $\phi 2$ & $\mathrm{~N} 39, \mathrm{~N} 37, \mathrm{~N} 78, \mathrm{~N} 77, \mathrm{~N} 09$ \\
$\gamma$ & $\gamma 1$ & $\mathrm{~N} 47, \mathrm{~N} 45$ \\
$\gamma$ & $\gamma 2$ & $\mathrm{~N} 75, \mathrm{~N} 51, \mathrm{~N} 49, \mathrm{~N} 30, \mathrm{~N} 07, \mathrm{~N} 06$ \\
$\eta$ & $\eta 1$ & $\mathrm{~N} 13, \mathrm{~N} 14, \mathrm{~N} 29, \mathrm{~N} 28, \mathrm{~N} 23, \mathrm{~N} 33, \mathrm{~N} 38, \mathrm{~N} 19, \mathrm{~N} 27, \mathrm{~N} 34, \mathrm{~N} 35, \mathrm{~N} 18, \mathrm{~N} 15, \mathrm{~N} 10$ \\
& $\eta 2$ & $\mathrm{~N} 24, \mathrm{~N} 22, \mathrm{~N} 32, \mathrm{~N} 31, \mathrm{~N} 21, \mathrm{~N} 03, \mathrm{~N} 04, \mathrm{~N} 02, \mathrm{~N} 08, \mathrm{~N} 20, \mathrm{~N} 01$ \\
\hline
\end{tabular}

The 40 yellow waxy maize inbred lines, except N12, were clustered into SSRG VI, SSRG VII, SSRG VIII, and SSRG IX; 28 yellow lines (93\%) belonged to SSRG IX, and the other 11 yellow lines were grouped into SSRG VI, SSRG VII and SSRG VIII, separately. The $21(84 \%)$ white inbred lines were clustered into SSRG III, SRG V, SSRG VI and the third subgroup of SSRG II. The white N69 line was solely grouped into the first subgroup of SSRG II. For the black inbred lines, N72 was clustered into SSRG IV of its own, and N75, N76, N78 were clustered into SSRG VII, SSRG VIII and SSRG IX, respectively. The other nine black lines $(57 \%)$ were clustered into SSRG II. The two red inbred lines were clustered into the second subgroup of SSRG II, and there was no difference at the level of GD (Table 4).

\section{DISCUSSION}

In this study, Nei's GD among the 80 waxy maize inbred lines were between 0.050 and 0.620 , which revealed that there were more differences between populations. The results of SSR amplification showed that 60 polymorphic alleles were amplified by 22 SSR primer pairs and that the polymorphic alleles ranged from 2 to 4 per locus, with an average of 2.73 . This finding demonstrated that SSR primers can detect the genetic differences between waxy maize inbred lines in this test.

The clustering results using the UPGMA method based on the GD showed that there were genetic differences between the four different color waxy maize inbred lines and that the GD within the same color inbred lines (the GD for the yellow ones, white ones and black ones was $0.632,0.681$ and 0.660 , respectively) was significantly less than that between the different color ones (the GD between the yellow and white ones, yellow and black ones, black and white ones was $0.746,0.753$ and 0.720 , respectively). There was more difference of the minimum GD between the different color ones than that of the same color ones (the minimum GD between the yellow and white ones, yellow and black ones, black and white ones was 0.464 , 0.517 and 0.483 , respectively). Between the yellow, white and black populations, the GD was $0.087,0.091$ and 0.045 , respectively. The above results were consistent with the findings of Fu (1995), who found that there was no significant difference between the different color inbred lines according to the genetic distance. 
Therefore, we concluded that it was more accurate to determine the difference between the populations using the highly stable DNA genetic markers. The results of SSR analysis also gave the better demonstration that the minimum genetic distance between the same color ones approached zero, which meant homogeneous propinquity. For instance, N34 and N35, N54 and N53, N77 and N78 were siblines from the same pedigree. Although the GD within the same phenotype was less than that between different ones, the variation range of the GD within the same phenotype cluster was larger. This suggests that there was a great potential difference within the same cluster. Therefore, it was possible to select the best inbred lines from N36 and N12, N69 and N72, and N49 and N65.

\section{ACKNOWLEDGMENTS}

Research supported by the Department of Science and Technology, Jilin Province, China (Project \#20080573).

\section{REFERENCES}

Fu TL (1995). The analysis of genetic principal component and distance of 33 glutinous maize inbred lines. Sci. Agric. Sin. 28: 46-53.

Liu YJ, Huang YB, Rong TZ, Tian ML, et al. (2005). Comparative analysis of genetic diversity in landraces of waxy maize from Yunnan and Guizhou using SSR markers. Sci. Agric. Sin. 4: 648-653.

Nei M and Li WH (1979). Mathematical model for studying genetic variation in terms of restriction endonucleases. Proc. Natl. Acad. Sci. U. S. A. 76: 5269-5273.

Novy RG, Vorsa N, Kobak C and Goffreda J (1994). RAPDs identify varietal misclassification and regional divergence in cranberry [Vaccinium macrocarpon (Ait.) Pursh]. Theor. Appl. Genet. 88: 1004-1010.

Reif JC, Melchinger AE, Xia XC, Warburton ML, et al. (2003a). Genetic distance based on simple sequence repeats and heterosis in tropical maize populations. Crop Sci. 43: 1275-1282.

Reif JC, Melchinger AE, Xia XC, Warburton ML, et al. (2003b). Use of SSRs for establishing heterotic groups in subtropical maize. Theor. Appl. Genet. 107: 947-957.

Senior ML, Murphy JP, Goodman MM and Stuber CW (1998). Utility of SSRs for determining genetic similarities and relationships in maize using an agarose gel system. Crop Sci. 38: 1088-1098.

Shehata AI, Al-Ghethar HA and Al-Homaidan AA (2009). Application of simple sequence repeat (SSR) markers for molecular diversity and heterozygosity analysis in maize inbred lines. Saudi J. Biol. Sci. 16: 57-62.

Smith JSC and Weissinger H (1984). Rapid monitoring of purity in seed lots of hybrid maize: modifications of current technologies. Maize Genet. Coop. Newslett. 2: 103-105.

Smith JSC, Chin ECL, Shu H, Smith OS, et al. (1997). An evaluation of the utility of SSR loci as molecular markers in maize (Zea mays): Comparisons with data from RFLPs and pedigree. Theor. Appl. Genet. 95: 163-173.

Wang C, Bian K, Zhang H-X and Zhou Z-M (1994). Polyacrylamide gel electrophoresis of salt-soluble proteins for maize variety identification and genetic purity assessment. Seed Sci. Technol. 22: 51-57.

Wu MS, Dai JR and Wang SC (1999). Application of RAPD in cultivar identification and purity test in maize. Acta Agron. Sin. 25: 489-493.

Xia XC, Hoisington DA and Warburton ML (2004). Genetic diversity among CIMMYT maize inbred lines investigated with SSR markers: I. Lowland tropical maize. Crop Sci. 44: 2230-2237.

Yao Q, Yang K, Pan G and Rong T (2007). Genetic diversity of maize (Zea mays L.) landraces from southwest China based on SSR data. J. Genet. Genomics 34: 851-859. 